\title{
Author Index Vol. 44, 1981
}

$(\mathrm{A})=$ Abstracts

Amano, K. (A) 377 Andreesen, W.H. 30 Asano, T. (A) 380

Bertrand, G. 302 Bowcock, S.A. 62 Broggi, G. 77

Campbell, J.N. 181,207 Campos, R.J. 119, 141 Cook, A.W. 55 Cooke, E.D. 62 Crenna, P. 171

Davis, R. 37, 160 Delwaide, P.J. 171 Dieckmann, G. 291 Dimitrijevic, M.M. 119, 141 Dimitrijevic, M.R. 119,

133, 152 Doi.A. (A) 376 Dooley, D.M. 71,218

Eccles, J.C. 5

Eguchi, T. (A) 374 Emson, P.C. 62 Erickson, D. 207

Faganel, J. 133, 141 Fawcett, D. 62 Feger, J. (A) 381 Friedman, H. 225 Fujimoto, N. (A) 375 Fukami, T. (A) 378 Furui, T. (A) 377

Gildenberg, P.L. 233 Girgis, M. 338 Gray, E. 37, 160 Grimes, J. 225 
Hammond, C. (A) 381 Hanamura, T. (A) 373,

374 Hardy, T.L. 302 Hassler, R. 291 Hawkes, C.H. 62 Hirai, T. (A) 379 Hondo, H.

(A) 375 Hood,T.W. 314

Iai, S. (A) 374 Imamura, Y. (A) 371 Imura, H. (A) 377 Iseki, H. (A) 377 Iwabuchi, T. (A) 371 Iwata, K. (A) 374,375

Jenker, F.L. 330 Job, I. 355

Kageyama, N. (A) 374,

375, 377, 379 Katayama, Y. (A) 372 Kawabatake, H. (A) 377 Kawamura, H. (A) 377 Kitamura, K. (A) 371, 377 Komai, N. (A) 376 Kowada, M. (A) 374 Kudzma, J. 37

Larsson, L.-E. 152 Lazorthes, Y. 77 LeRoy, P.L. 187 Levi, F.P. 338 Levita, E. 93 Long, D.M. 207

Matsumoto, K. (A) 375,

378 Matsumoto, Y. (A) 380 Mayanagi, Y. (A) 373, 374 
Mehler.W.R. 261 Meyer, C.H.A. 345 Mingrino, S. 355 Moriwaki, H. (A) 376 Moriyasu, N. (A) 372 Murayama, Y. (A) 378 Murthy, K.S.K. 22 Mutsuga, N. (A) $374,377,379$

Nagao, T. (A) 377 Nagaseki, Y. (A) 379 Nakano, H. (A) 374 Nakao, K. (A) 377 Nakao, Y. (A) 380 Nakaoka, T. (A) 371 Nashold, B., Jr. 225 Nidzgorski, F. 55 Nishiguchi, T. (A) 376 Nishimoto, A. (A) 380 Nishimoto, H. (A) 372 Nisonson, I. 71 North, R. 207 Notani, M. (A) 377

Oda, N. (A) 371 Ogura, K. (A) 379 Ohmoto, T. (A) 380 Ohye, C. (A) 379 Okada, T. (A) 379

Paul, E.A. 62 Phillips, C.G. 16

Ray, CD. 194 Reynolds, L.O. 244 Riklan, M. 244 Rilan, M. 93

Saito, T. (A) 374 Sakamoto, M. (A) 378 
Author Index 
Sakamoto, T. (A) 374 Sakurai, M. (A) 380 Salar, G. 355 Schuhfried, F. 330 Sharkey, J. 218 Sharkey, P.C. 50, 119, 141 Sherwood, A.M. 126 Shibazaki, T. (A) 379,381 Shibuya, M. (A) 379 Shichijo, F. (A) 378 Shima, F. (A) 371 Shiwaku, T. (A) 377 Siegfried, J. 77 Smith, J. 363 Soga, T. (A) 375 Sogabe, K. (A) 375,378 Stejskal, L. 320 
Struppler, A. 114 Struppler, E. 114 Suzuki, I. (A) 373, 374 Suzuki, Y. (A) 379

Tanikawa, T. (A) 377 Taylor, J.K. 55 Thompson, C.J. 302 Tomanek, Z. 320

Tosaki, F. (A) 374, 375 Toulouse, P. 171 Tsubokawa, T. (A) 372 Tsuda, T. (A) 378

Vasilescu, C. 291 Vladyka, V. 320 Vodovnik, L. 97 
Waltz, J.M. 30, 93, 244 Watanabe, E. (A) 374 Watanabe, S. (A) 375

Yamamoto, T. (A) 372 Yamashita, S. (A) 375 Yap, J.C. 314 Yoneya, M. (A) 374

Yoshimasa, T. (A) 377 Young, R.R. 133 Yuasa, H. (A) 374, 375 